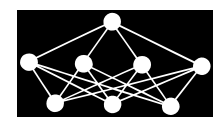

\title{
WAVE COMPOSITION RULES IN QUANTUM SYSTEM THEORY
}

\author{
Miroslav Svítek*
}

\begin{abstract}
The paper presents the new approach to wave composition rules for advanced modeling of soft systems in quantum system theory. Firstly, the interpretation of phase parameters is given. The phase parameters are essential to specify the mathematical operations assigned to different relations among subsystems, e.g. co-operation, connection, co-existence, competition. Using wave composition rules, we are able to create more complex and sophisticated quantum circuits. We present the application of methodology on three illustrative examples.
\end{abstract}

Key words: quantum system theory, quantum information systems, information power, information physics, self-organization, composition rules

Received: January 23, 2019

DOI: $10.14311 /$ NNW.2020.30.004

Revised and accepted: February 19, 2020

\section{Introduction}

Quantum system theory $[1,13]$ describes the environmental properties as the phase space of interconnected events. The entanglement of quantum states leads to spacetime synergies between particular events, which can be considered as a form of ordering [14]. Knowing 'something' means knowing what 'something' transcends - this feature is modeled using phase parameters. Naturally, everything is a part of its inherent environment and nothing can be larger than its own environment. Monitoring of unrealized possibilities and opportunities in our environment is exploring its major part. What has not happened is a necessary complement to what has happened, and it is important to realize that it is sometimes more important than what has happened.

The inspiration of how to understand environment modeling together with its composition rules came from the reflection theory of waves of a long power line and telegraph line [12]. We can recognize the non-end termination (infinite resistance), short-circuit termination (zero resistance) or termination with given impedance. Physics explains that non-end termination yields into wave reflection with an opposite phase (a reflected wave is shifted through 180 degrees). After interference, it causes standing waves. Short-circuit termination causes a reflection with the same phase (no phase shift of reflected wave). If any impedance terminates a power line,

\footnotetext{
* Miroslav Svítek; Czech Technical University in Prague, Faculty of Transportation Sciences, Konviktská 20, Prague 1, Czech Republic, E-mail: svitek@fd.cvut.cz; Matej Bel University, Faculty of Natural Sciences, Tajovského 40, Banská Bystrica, Slovak Republic
} 
the reflected wave depends on the impedance's characteristics and the interference with the original wave can be computed using the theory of electrical circuits [8].

Section 2 presents the author's approach to the interpretation of wave probabilistic functions. Section 3 extends such approach to different system's structures and describes the wave composition rules for different operations with probabilistic wave functions. This methodology is appropriate for modeling of soft systems. Section 4 presents the symmetric interactions of co-operating or competing subsystems. In Section 5, this approach is applied to model interaction between a subsystem and its environment. Illustrative examples of possible applications are given in Section 6. Section 7 concludes the paper.

\section{Interpretation of wave probabilistic functions}

Let us define the set of $N$ basic subsystems with wave representations [1]:

$$
\psi_{S_{i}}=M_{S_{i}} \cdot \mathrm{e}^{j \cdot \varphi_{S_{i}}} \quad i \in\{1,2, \ldots, N\} .
$$

Modulus $M_{S_{i}}=\sqrt{p_{S_{i}}}$ represents the square of probability $p_{S_{i}}$ (the dominance or strength of the subsystem $S_{i}$ ) and $\varphi_{S_{i}}$ is the phase parameter (the ability to co-operate or being incorporated into the system as a whole).

\subsection{Phase parameters in quantum subsystems}

For the sake of simplicity, we suppose constant modules $M_{i, k}$ and a phase dynamics of components $\nu_{i, k}$ in the following way:

$$
\psi_{S_{i}}(t)=\sum_{k=1}^{N} M_{i, k} \cdot \mathrm{e}^{j \cdot \nu_{i, k} \cdot t}
$$

In signal processing, $\nu$ - components represent harmonic frequencies obtained by Fourier transform [2]. In quantum system theory, we can interpret $\nu_{i, k}$ as the "data flow rate" of $i$-th subsystem in [bits/s]. In the case $\nu_{i, k}$ is higher, the $k$-th component of $i$-th subsystem is able to exchange more data per second with its environment.

Assume further that we can distinguish between positive and negative data flow rates $\nu$ that means a clockwise or anticlockwise rotation in phase space. Let us agree an assumption within this theory that positive values mean ordering. In contrast, negative values represent disordering (chaotisation). Connecting the same value of order and disorder yields to zero phase. If the data flow rate is zero the subsystem interacts only randomly without any sophisticated structure of dependencies. Maximal value of data flow rate $\nu_{\max }$ specifies a threshold of communication possibilities of the subsystem.

\subsection{Phase parameters in quantum circuits}

Quantum circuit is characterized by a wave information flow $\Phi$ and a wave information content $I$ introduced in [10] as wave probabilistic functions. For simplicity, 
we use only simple phase dynamics of data flow rates $\nu_{\Phi}$ and $\nu_{I}$ :

$$
\psi_{\Phi}(t)=\left|\psi_{\Phi}(t)\right| \cdot \mathrm{e}^{j \cdot\left(\nu_{\Phi} \cdot t+\varphi_{\Phi}\right)}, \quad \psi_{I}(t)=\left|\psi_{I}(t)\right| \cdot \mathrm{e}^{j \cdot\left(\nu_{I} \cdot t+\varphi_{I}\right)} .
$$

Phases $\varphi_{\phi}$ and $\varphi_{I}$ represent static interactions (organization of experiment) and $\nu_{\Phi}$ and $\nu_{I}$ dynamic data exchange among circuits components. For quantum system modeling, the phase difference between $\Phi$ and $I$ is important to compute an active and a reactive information power [3].

\subsection{Phase parameters in quantum physics}

For physical interpretation of phase parameters, we get the inspiration in a holographic approach described in $[4,5]$. Suppose the real observation relief (an objective reality) is specified as:

$$
s(x)=A+B \cdot \cos \left(\omega_{x} \cdot x\right) .
$$

Value $A$ is a constant signal and $B$ is the amplitude of cosine changes in $x$-axis with frequency $\omega_{x}$. Now, suppose the observer uses a cosine time signal $\cos \left(\omega_{t} \cdot t\right)$ with wavelength $\lambda_{t}$. If the observer is located directly at a point $x$, it watches the complex signal where the objective reality is modulated on light waves:

$$
\psi(x, t)=\left[A+B \cdot \cos \left(\omega_{x} \cdot x\right)\right] \cdot \mathrm{e}^{j \cdot \omega_{t} \cdot t} .
$$

In a case the observer is watching the scene from a distance $z$, we must take into account the diffraction rules. The image of reality seen from a distance $z$ (observed signal) can be calculated using the Fresnel-Kirchhoff diffraction integral [6]. For our simplified relief (4), the observer watches:

$$
\psi_{z}(x, t)=A \cdot \mathrm{e}^{j \cdot \omega_{t} \cdot t}+\frac{1}{2} \cdot B \cdot\left(\mathrm{e}^{j \cdot\left(\omega_{t} \cdot t+\omega_{x} \cdot x+\Phi_{z}\right)}+\mathrm{e}^{j \cdot\left(\omega_{t} \cdot t-\omega_{x} \cdot x+\Phi_{z}\right)}\right) .
$$

The new phase parameter can be computed from the geometry [7]:

$$
\Phi_{z}=\frac{1}{2 \cdot \pi} \cdot \omega_{x}^{2} \cdot \lambda_{t} \cdot z
$$

In a real observation process, the phase parameters are not available to us. We can measure only the probability or energy spectrum:

$$
\begin{aligned}
& P_{z}=\psi_{z}(x, t) \cdot \psi_{z}^{*}(x, t)=A^{2}+2 \cdot A \cdot B \cdot \cos \left(\Phi_{z}\right) \cdot \cos \left(\omega_{x} \cdot x\right)+ \\
& +\frac{1}{2} \cdot B^{2} \cdot \cos \left(2 \cdot \omega_{x} \cdot x\right)+\frac{1}{2} \cdot B^{2} .
\end{aligned}
$$

By omitting the distortion components (e.g. higher frequencies), we can extract useful information from the reality observed:

$$
P_{z} \approx A^{2}+2 \cdot A \cdot B \cdot \cos \left(\Phi_{z}\right) \cdot \cos \left(\omega_{x} \cdot x\right) .
$$

From the above equation it is evident how the phase parameter $\Phi_{z}$ can bias an observation process. For better clarification, we can rewrite equation (6) in a "bracket" notation [8]:

$$
\psi_{z}(x, t) \propto A \cdot|1\rangle+B \cdot e^{j \cdot \Phi_{z}}\left|\cos \left(\omega_{x} \cdot x\right)\right\rangle .
$$


Intersection operation represents the coexistence of constant and cosine signals together.

The phase parameter $\Phi_{z}$ in our approach defines the quality of the observation process, how the image of reality is distorted due to imperfect observations. Wave nature disappears if the phase is zero. It can occur if the observer is located directly at the place of measurement $(z \approx 0)$, or if the measurement is done with infinite resolution $\left(\lambda_{t} \approx 0\right)$. Unfortunately, the measurements in quantum physics are limited by Planck distance and wavelength of light [11] so the phase cannot be zero.

\section{Wave composition rules}

\subsection{Connected subsystems}

Let us introduce two dynamical subsystems described by wave probabilistic functions:

$$
\psi_{S_{1}}(t)=M_{S_{1}} \cdot \mathrm{e}^{j \cdot \nu_{1} \cdot t}, \psi_{S_{2}}(t)=M_{S_{2}} \cdot \mathrm{e}^{j \cdot \nu_{2} \cdot t}
$$

The connection means that both subsystems are firmly connected in one system and together order the near environment. This situation is common in quantum physics and it is generally represented by the Kronecker operation [9] for vectors transformed into multiplication. We can thus provide the following operation for connected quantum subsystems:

$$
\psi_{S_{1,2}}(t)=\psi_{S_{1}}(t) \cdot \psi_{S_{2}}(t)=M_{S_{1}} \cdot M_{S_{2}} \cdot \mathrm{e}^{j \cdot\left(\nu_{1}+\nu_{2}\right) \cdot t} .
$$

For this case, phase parameters $\nu_{1}, \nu_{2}$ are added. In analogy with a power line, it is a short-cut connection with zero resistance.

\subsection{Co-operated subsystems}

The co-operation in quantum system theory means that both subsystems are encapsulated inside a superior system without firm connection. In other words, they are connected with infinite resistance (for a power line, it is the same as a non-end connection).

We suppose both subsystems can exchange data. Imagine that the subsystem $S_{1}$ is an active one and sends its request to a passive subsystem $S_{2}$. Subsystem $S_{2}$ replies with an opposite phase function - it actually replies the environment of $S_{2}$, which has an opposite phase. From the point of view of $S_{1}$ the received data is a colored reflection of subsystem $S_{2}$. Wave representation of such operation can be written:

$$
\psi_{S_{1,2}}(t)=\psi_{S_{1}}(t) \cdot \psi_{S_{2}}^{*}(t)=M_{S_{1}} \cdot M_{S_{2}} \cdot \mathrm{e}^{j \cdot\left(\nu_{1}-\nu_{2}\right) \cdot t} .
$$

The co-operated rules for $S_{1}$ yields into the phases $\nu_{1}, \nu_{2}$ subtraction. If the activity comes from subsystem $S_{2}$ we can speak about co-operated rules for $S_{2}$ with final phase $\left(\nu_{2}-\nu_{1}\right)$. 


\subsection{Co-existed subsystems}

Co-existence in quantum system theory represents the separate existence of both subsystems $S_{1}$ and $S_{2}$ in one environment. The co-existence is expressed by sum of wave functions:

$$
\psi_{S_{1,2}}(t)=\psi_{S_{1}}(t)+\psi_{S_{2}}(t)=M_{S_{1}} \cdot \mathrm{e}^{j \cdot \nu_{1} \cdot t}+M_{S_{2}} \cdot \mathrm{e}^{j \cdot \nu_{2} \cdot t}
$$

A union of wave functions describes how we can work with the subsystems $S_{1}$ and $S_{2}$ separately, or with both of them at the same time.

\section{Symmetric interactions}

\subsection{Symmetrically co-operative subsystems}

We expect that each subsystem is able both to send and receive data from the second one. If the first subsystem $S_{1}$ starts the data transfer and the second $S_{2}$ replies we can apply the rule of co-operated subsystems:

$$
\psi_{S, 1}(t)=\psi_{S_{1}}(t) \cdot \psi_{S_{2}}^{*}(t)=M_{S_{1}} \cdot M_{S_{2}} \cdot \mathrm{e}^{j \cdot\left(\nu_{1}-\nu_{2}\right) \cdot t} \cdot
$$

Conversely, if the second subsystem $S_{2}$ initiates communication and the first subsystem $S_{1}$ sends its reply we have wave function:

$$
\psi_{S, 2}(t)=\psi_{S_{2}}(t) \cdot \psi_{S_{1}}^{*}(t)=M_{S_{1}} \cdot M_{S_{2}} \cdot \mathrm{e}^{-j \cdot\left(\nu_{1}-\nu_{2}\right) \cdot t}
$$

Because both variants are equal (symmetric interaction), we can express the final waveform as a co-existence of both variants:

$$
\psi_{S}(t)=\psi_{S_{1}}(t) \cdot \psi_{S_{2}}^{*}(t)+\psi_{S_{1}}^{*}(t) \cdot \psi_{S_{2}}(t)=2 \cdot M_{S_{1}} \cdot M_{S_{2}} \cdot \cos \left(\left(\nu_{2}-\nu_{1}\right) \cdot t\right)
$$

In quantum physics, we speak about indistinguishable subsystems because both have the same option to communicate. The result can be interpreted as a periodical exchange of a common probability/energy value $M_{S_{1}} \cdot M_{S_{2}}$ between both subsystems $S_{1}$ and $S_{2}$. This principle is called in quantum physics an exchange of a virtual particle.

\subsection{Symmetrically competing subsystems}

Sometimes, there is only one state for only one subsystem. If two subsystems start their competition, only one of them can be the winner (winner takes all). This situation can modify the rule for wave probabilistic function in the following way:

$$
\psi_{S}(t)=\psi_{S_{1}}(t) \cdot \psi_{S_{2}}^{*}(t)-\psi_{S_{1}}^{*}(t) \cdot \psi_{S_{2}}(t)
$$

In quantum physics, it yields to the well-known Pauli Exclusion Principle. 


\section{Interactions with an environment}

In this session, we suppose that only one subsystem $S_{1}$ exists within the environment $S_{E}$. If the environment is closed (not connected to other systems) then the energy (probability) conversation law must be fulfilled - the energy (probability) of subsystem $S_{1}$ must be equal to $M_{S_{1}}^{2}$. It means that environment $S_{E}$ must have an opposite phase (a mirror image of the subsystem $S_{1}$ ):

$$
\psi_{S_{E}}(t)=M_{S_{1}} \cdot \mathrm{e}^{-j \cdot \nu_{1} \cdot t}
$$

Because the environment $S_{E}$ is connected with subsystem $S_{1}$ we can write the final wave probabilistic operation between the subsystem $S_{1}$ and its environment $S_{E}$ as:

$$
\psi_{S, E}=\psi_{S_{1}}(t) \cdot \psi_{S_{E}}(t)=M_{S_{1}}^{2}
$$

We can imagine that subsystem $S_{1}$ organizes itself (positive phase) at the expense of its surroundings $S_{E}$, which it chaotizes (negative phase).

For more complicated connections among subsystems, the environment copies these structures to guarantee that the whole energy (probability) is conserved. All complicated links eventually lead to the resulting wave function, which has its amplitude and phase to which the environment reacts.

\section{Illustrative examples}

\subsection{Wave composition rules}

Let us define three subsystems $S_{1}, S_{2}, S_{3}$ with their wave probabilistic functions given by:

$$
\begin{gathered}
\psi_{S_{1}}=\alpha_{0}|0\rangle_{1}+\alpha_{1}|1\rangle_{1}, \\
\psi_{S_{2}}=\beta_{0}|0\rangle_{2}+\beta_{1}|1\rangle_{2}+\beta_{2}|2\rangle_{2}, \\
\psi_{S_{3}}=\gamma_{0}|0\rangle_{3}+\gamma_{1}|1\rangle_{3} .
\end{gathered}
$$

Symbol $|k\rangle_{i}$ means the state $k$ falling on the subsystem $S_{i}$.

We suppose that $S_{1}$ and $S_{2}$ are firmly connected in $S_{1,2}$ that is represented by wave function:

$$
\begin{aligned}
& \psi_{S_{1,2}}=\alpha_{0} \cdot \beta_{0}|00\rangle_{1,2}+\alpha_{0} \cdot \beta_{1}|01\rangle_{1,2}+\alpha_{0} \cdot \beta_{2}|02\rangle_{1,2}+ \\
& +\alpha_{1} \cdot \beta_{0}|10\rangle_{1,2}+\alpha_{1} \cdot \beta_{1}|11\rangle_{1,2}+\alpha_{1} \cdot \beta_{2}|12\rangle_{1,2}
\end{aligned}
$$

Both of them co-exist with subsystem $S_{3}$ in a common environment. This situation can be formulated:

$$
\begin{aligned}
& \psi_{S_{1,2,3}}=\alpha_{0} \cdot \beta_{0}|00\rangle_{1,2}+\alpha_{0} \cdot \beta_{1}|01\rangle_{1,2}+\alpha_{0} \cdot \beta_{2}|02\rangle_{1,2}+ \\
& +\alpha_{1} \cdot \beta_{0}|10\rangle_{1,2}+\alpha_{1} \cdot \beta_{1}|11\rangle_{1,2}+\alpha_{1} \cdot \beta_{2}|12\rangle_{1,2}+\gamma_{0}|0\rangle_{3}+\gamma_{1}|1\rangle_{3} .
\end{aligned}
$$


After interacting with the environment, we obtain the following energies (probabilities) assigned to all possible variants of the final outputs:

$$
\begin{aligned}
& P_{1,2,3}=\psi_{S_{1,2,3}} \cdot \psi_{S_{1,2,3}}^{*}=p_{00}|00\rangle_{1,2}+p_{01}|01\rangle_{1,2}+p_{02}|02\rangle_{1,2}+p_{10}|10\rangle_{1,2}+ \\
& +p_{11}|11\rangle_{1,2}+p_{12}|12\rangle_{1,2}+p_{000}|000\rangle_{1,2,3}+p_{010}|010\rangle_{1,2,3}+p_{020}|020\rangle_{1,2,3}+ \\
& +p_{100}|100\rangle_{1,2,3}+p_{110}|110\rangle_{1,2,3}+p_{120}|120\rangle_{1,2,3}+p_{001}|001\rangle_{1,2,3}+p_{011}|011\rangle_{1,2,3} \\
& +p_{021}|021\rangle_{1,2,3}+p_{101}|101\rangle_{1,2,3}+p_{111}|111\rangle_{1,2,3}+p_{121}|121\rangle_{1,2,3}
\end{aligned}
$$

For the above computing we used logical operations [14]:

$$
\begin{gathered}
\alpha_{0} \cdot \beta_{0} \cdot|00\rangle_{1,2} \cdot \alpha_{0}^{*} \cdot \beta_{1}^{*} \cdot|01\rangle_{1,2}=\frac{1}{2} \cdot \alpha_{0} \cdot \beta_{0} \cdot \alpha_{0}^{*} \cdot \beta_{1}^{*} \cdot\left(|00\rangle_{1,2}+|01\rangle_{1,2}\right), \\
\alpha_{0} \cdot \beta_{0} \cdot|00\rangle_{1,2} \cdot \gamma_{0}^{*} \cdot|0\rangle_{3}=\alpha_{0} \cdot \beta_{0} \cdot \gamma_{0}^{*} \cdot|000\rangle_{1,2,3} \cdot
\end{gathered}
$$

By using an intersection or a union operation, we can compute different probabilities, for example:

- $|00\rangle_{1,2} \mathrm{OR}|000\rangle_{1,2,3}$ is $p_{00}+p_{000}$

$$
\text { - }\left(|00\rangle_{1,2} \text { OR }|000\rangle_{1,2,3}\right) \text { AND }|10\rangle_{1,2} \text { is }\left(p_{00}+p_{000}\right) \cdot p_{10}
$$

The above example shows how we can compute the final energy (probability) for specified structures of the system [10] with different links (connection, co-operation, co-existence) among the components / subsystems.

\subsection{Real and observed reality}

Let us introduce two extended descriptions of dynamical subsystems (11):

$$
\psi_{S_{1}, O_{k}}(t)=M_{S_{1}} \cdot \mathrm{e}^{j \cdot\left(\nu_{1} \cdot t+\Phi_{1, O_{k}}\right)}, \psi_{S_{2}, O_{k}}(t)=M_{S_{2}} \cdot \mathrm{e}^{j \cdot\left(\nu_{2} \cdot t+\Phi_{2, O_{k}}\right)} .
$$

Added phase parameters $\Phi_{1, O_{k}}$ and $\Phi_{2, O_{k}}$ represent the phase shifts. In other words, they explain how $k$-observer perceives the first and the second subsystem from its subjective point of view. If these phases are zeros we can speak about the real reality (without any observation error).

For $M$ observers we have at disposal $M$ images of observed reality assigned to each observation process. This situation represents a relativity of a perceived reality. The question in this example is how to approximate the real reality in the best way? We can apply a democratic principle and use the average of all observations:

$$
\begin{aligned}
& \bar{\psi}_{S_{1}}(t)=M_{S_{1}} \cdot \mathrm{e}^{j \cdot \nu_{1} \cdot t} \cdot \frac{1}{M} \cdot \sum_{k=1}^{M} \mathrm{e}^{j \cdot \Phi_{1, O_{k}}}=\bar{M}_{S_{1}} \cdot \mathrm{e}^{j \cdot\left(\nu_{1} \cdot t+\bar{\Phi}_{1}\right)}, \\
& \bar{\psi}_{S_{2}}(t)=M_{S_{2}} \cdot \mathrm{e}^{j \cdot \nu_{2} \cdot t} \cdot \frac{1}{M} \cdot \sum_{k=1}^{M} \mathrm{e}^{j \cdot \Phi_{2, O_{k}}}=\bar{M}_{S_{2}} \cdot \mathrm{e}^{j \cdot\left(\nu_{2} \cdot t+\bar{\Phi}_{2}\right)},
\end{aligned}
$$

The final values $\bar{M}_{S_{1}}, \bar{\Phi}_{1}, \bar{M}_{S_{2}}, \bar{\Phi}_{2}$ can be taken as the best approximation of the reality. 
It is evident that the illustrative example can be extended into systems with more components (subsystems) and more sophisticated dynamical links among them (connection, co-existence, co-operation, competition, etc.). We can model through the presented methodology also the time varying observations, taking into account both learning and forgetting processes.

\subsection{Different relationships among subsystems}

We can generalize the previous example and extend it to a set of $N$ different subsystems with their own relations to other subsystems. For the sake of simplicity, we assume that modulus $M_{s_{i}}$ assigned to $i$-th subsystem is the same for all links. Wave function of multi-links $i$-th subsystem can be given in matrix form:

$$
\psi_{S_{i}}=M_{S_{i}} \cdot\left[\begin{array}{c}
\mathrm{e}^{j \cdot \Phi_{i, 1}} \\
\mathrm{e}^{j \cdot \Phi_{i, 2}} \\
\mathrm{e}^{j \cdot \dot{\Phi}_{i, N}}
\end{array}\right]
$$

The phase $\Phi_{i, k}$ is parameter explaining how $i$-th subsystem perceives the $k$-th one. Phase $\Phi_{i, i}$ describes how $i$-th subsystem sees itself in relation with common reference. Co-existence of $\mathrm{N}$ subsystems in an environment can be written by wave function:

$\psi_{S}=M_{S_{1}} \cdot\left[\begin{array}{c}\mathrm{e}^{j \cdot \Phi_{1,1}} \\ \mathrm{e}^{j \cdot \Phi_{1,2}} \\ \mathrm{e}^{j \cdot \dot{\Phi}_{1, N}}\end{array}\right] \cdot\left|S_{1}\right\rangle+M_{S_{2}} \cdot\left[\begin{array}{c}\mathrm{e}^{j \cdot \Phi_{2,1}} \\ \mathrm{e}^{j \cdot \Phi_{2,2}} \\ \mathrm{e}^{j \cdot \Phi_{2, N}}\end{array}\right] \cdot\left|S_{2}\right\rangle+\cdots+M_{S_{N}} \cdot\left[\begin{array}{c}\mathrm{e}^{j \cdot \Phi_{N, 1}} \\ \mathrm{e}^{j \cdot \Phi_{N, 2}} \\ \mathrm{e}^{j \cdot \Phi_{N, N}}\end{array}\right] \cdot\left|S_{N}\right\rangle$.

By the use of matrix representation, we can provide all manipulations (union, intersection, etc.) in more dimensional space. It is visible that each phase parameter $\Phi_{i, k}$ carry useful information. These phase parameters could be time varying and information can be modulated on these carriers.

\section{Conclusion}

The inspiration of this paper originated in the reflection theory on a long power and telegraph line [12]. This approach was modified for phase parameters used in quantum system theory [1]. With respect to the physics/information analogies [13], different kinds of terminations have been replaced by characteristics of linked subsystems and by characteristics of the environment.

Wave function with opposite phase is a supplement of the subsystem. Connection of the subsystems with its environment leads to a zero phase that means a fully probabilistic nature - pure energy without any sophisticated organization. The main goal of an arranging process is to transform a pure energy (zero phase) into a more sophisticated one at the expense of the energy of environment. If we organize our environment, we can obtain from the energy difference between disorderliness and order the energy source of life. It is clear that living organisms communicate with their environment to benefit from it [15]. 
For practical use of the quantum system theory [1], it was necessary to develop the wave composition rules, how the different links among subsystems could be modeled. We identified the different relations among subsystems like connection, co-existence, co-operation, symmetric co-operation and symmetric competition.

Finally, we presented three examples to illustrate the practical applicability of our approach. The first example shows the composition rules of three discrete quantum subsystems. Common connection and co-existence of the subsystems were analyzed and the wave composition rules were applied to compute final probabilities. The second example simulates a set of many observers with different point of views on real reality modeled by shifted phase parameters. Quantum system theory can provide us with approximate best result through a well-known democratic approach. This view is naturally characterized by both modulus and phase of wave probabilistic function. What we call the truth is often the result of some social agreement or even a consensus approximated by the average of different subjective wave functions. The third example extends this approach to different relationships to other subsystems that reflects its own perception of each subsystem. It is possible to model reality while respecting the views of all subsystems.

There is a view that a world is composed of miniature, quantum, in some ways illusory worlds. We create our world by how we think, what we focus on and how we behave and act. The own worlds of the various observers (wave functions) interact with each other and create a common information field that then represents the complex knowledge through which our world is conveyed to us.

\section{Acknowledgement}

This work was supported by the Project AI \& Reasoning CZ.02.1.01/0.0/0.0/15_003 /0000466 and the European Regional Development Fund.

\section{References}

[1] SVÍTEK M. Quantum System Modelling, International Journal on General Systems, 2008, 37(5), pp. 603-626, doi: 10.1080/03081070701833039.

[2] SHANNON C.E. A mathematical theory of communication, Bell Syst. Tech. J., 1948, 27, 379, 623, doi: $10.1002 / j \cdot 1538-7305.1948 . t b 00917 . x$.

[3] SVÍTEK M. Wave probabilistic information power, Neural Network World, 2011, 21(3), pp. 269-276, ISSN 1210-0552, doi: 10.14311/NNW.2011.21.016.

[4] KOGELNIK H. Coupled wave theory for thick hologram gratings. The Bell System Technical Journal, 1969, 48(9), 2909, doi: 10.1002/j.1538-7305.1969.tb01198.x.

[5] SAXBY G. Practical Holography. Prentice-Hall, ISBN, 1988, doi: 10.1887/0750309121.

[6] VEST C. M. Holographic Interferometry. John Wiley and Sons, 1979, doi: 10.1063/1. 2995245.

[7] LEITH E. N., UPATNIEKS J. Reconstructed Wavefronts and Communication Theory, Journal of the Optical Society of America, 1962, 52(10), pp. 1123-1130, doi: 10.1364/JOSA.52. 001123.

[8] FEYNMAN R., LEIGHTON R., SANDS M. Feynman lectures of physics, Addison Wesley Longman, Inc., USA, 1966.

[9] SVÍTEK M. Complementary variables and its application in statistics, Neural network World, 2007, pp. 237-253, ISSN 1210-0552. 


\section{Neural Network World 1/2020, 55-64}

[10] SVÍTEK M., VOTRUBA, Z., MOOS, P. Towards Information Circuits, Neural Network World, 2010, 20(2), pp. 241-247, ISSN 1210-0552.

[11] SVÍTEK M. Investigation to Heisenberg's uncertainty limit, Neural Network World, 2008, 18(6), pp. 489-498.

[12] KOThaRI D. P., NAGRATH I. J. Power System Engineering, Tata McGraw-Hill Eduacation, 2007.

[13] SVÍTEK M. Physics-Information Analogies, Neural Network World, 2018, 28(6), pp. 535550, doi: 10.14311/NNW.2018.28.030.

[14] SVÍTEK M. Quantum multidimensional models of complex systems, Neural Network World, 2019, 29(5), pp. 363-371, doi: 10.14311/NNW.2019.29.022.

[15] KAUfFMAN S. Autonomous Agents. In: John D. Barrow, P.C.W. Davies, and C.L. Harper Jr., eds., Science and Ultimate Reality: Quantum Theory, Cosmology, and Complexity, Cambridge University Press, 2004, doi: 10.1017/CB09780511814990.032. 